\title{
Unbalanced Growth in a Semi-continuous Culture System Designed for the Synchronization of Cell Division
}

\author{
By G. D. ANAGNOSTOPOULOS \\ Microbiology Department, Queen Elizabeth College, \\ University of London, Campden Hill, London W.8
}

(Accepted for publication 3 November 1970)

SUMMARY

Synchronization of division of Escherichia coli was induced in a semicontinuous culture system. A small-scale apparatus designed for the automatic operation of the system is described. The carbon (glucose)-limited defined medium was supplied at intervals of $\mathrm{I}$ to $4 \mathrm{~h}$. and half of the diluted culture was discharged. After a number of cycles the glucose supplied each time was just sufficient for the doubling of the 'self-established' population. A logarithmic loss of viability occurred at the beginning of each cycle in the presence of fresh medium, and it was thought that part of the population underwent unbalanced growth. This phenomenon and the observation that the viable population consisted of two equal parts dividing synchronously but at different times were thought to reflect a post-division unequal competence of the emerging two halves of the population to cope with the active growth induced by the incoming fresh medium.

\section{INTRODUCTION}

Bacterial cultures in the stationary phase of growth often show some degree of division synchrony when they are transferred into fresh medium.

Cutler \& Evans (1966) synchronized cultures of Escherichia coli and Proteus vulgaris by repeated transfer from the early stationary phase into fresh medium. To explain this, they suggested that the generally unfavourable growth conditions, in cultures of high cell density, inhibit cells at a particular stage in their division cycle. Following nucleic acid and protein analyses they claimed that their synchronizing method 'may be unique in producing cultures that grow both in cellular division and in genome synchrony'.

Nishi, Okamura \& Yanagita (1967) investigated the shift of cell-age distribution pattern occurring in aged cultures of Escherichia coli in an attempt to explain synchronization previously observed in the transition period between the lag and logarithmic phases. They ruled out, experimentally, the possibility that toxic substances accumulated during growth exerted a growth-limiting effect. Furthermore, they suggested that the oxygen depletion caused the synchronization observed when succinate but not glucose was the carbon source. Basically of the same "transition period' principle may be the phasing method employed by Dawson (1965). He adapted his cyclone column chemostat unit into a 'continuous phased growth' system. In this system the circulating culture is diluted periodically with an equal volume of fresh medium and half of the mixture is discharged. He claimed that a synchronization 
of cell division was achieved after about 20 to 30 such additions of fresh medium (Dawson \& Kurz, 1969). It was thought that if the total nutrients necessary for one doubling are supplied at one time to a synchronized population the cells should divide at the same time over the next doubling period, and if this was repeated at similar intervals the population should continue dividing synchronously. The question, then, is how does synchrony emerge? But no attempt was made to explain this.

Müller \& Dawson (1968), working with Candida utilis, concluded that cultures growing on a nitrogen-limited medium but not on a glucose-limited medium adjusted their growth rate to the dosing time imposed by the phasing technique. The degree of synchrony was increased from $65 \%$ with $4 \mathrm{~h}$. cycles to $80 \%$ with $\mathrm{I} 2 \mathrm{~h}$. cycles. Dawson \& Kurz (1969) reported doubling times of 4,6 and $8 \mathrm{~h}$. of a pseudomonad growing on a carbon-limited medium with phenylacetic acid as the sole source of carbon. Dawson (1965) made the claim, verified by Müller \& Dawson (1968), that the continuous phasing technique provides synchrony for an indefinite number of generations.

The method used by Dawson and his colleagues involved the use of a system designed to a capacity of $500 \mathrm{ml}$. In our work it was considered advantageous to design our phasing systems to a capacity of $50 \mathrm{ml}$., mainly with the following three objectives: (a) to establish a standard laboratory method for the supply of synchronized bacterial cultures; $(b)$ to study the forces which induce synchrony; and $(c)$ to study the response of cultures to changes in periodicity of feeding.

\section{METHODS}

Culture system. The apparatus described below was designed to a capacity of $50 \mathrm{ml}$. of culture/cycle. The continuously circulating culture is maintained in contact with a stream of sterile (filtered) air at a controlled flow rate. The growth temperature is maintained by circulating water through both the culture and medium vessels. The length of each cycle is determined by the inflow rate of an equal volume of fresh medium. After each addition of medium half of the mixture is discharged and a new growth cycle of the culture begins. The release of the fresh medium in $50 \mathrm{ml}$. quantities is the function of the siphon in the medium metering vessel, and the frequency of this is controlled by a time-switch connected with the medium pump, a Delta micrometering pump (Watson-Marlow Ltd, Marlow, Buckinghamshire).

The main parts of the system (Pl. I and Fig. I) comprise (i) the medium metering vessel; (ii) the air-pressure restoration vessel; (iii) the culture vessel; (iv) the culture pump.

(i) Medium metering vessel. This is an inverted $100 \mathrm{ml}$. chemostat developed by Pirt \& Callow (1964) for the culture of mammalian cells. The vessel is plugged with a rubber stopper through which four pieces of stainless-steel tube enter the vessel: (a) medium inlet, (b) air inlet, (c) a curved downwards air outlet (its opening is adjusted to the required highest medium level), $(d)$ the medium siphon; when the medium level rises close to the air outlet, the obstruction to the air outflow triggers the siphoning action and the immediate release of the $50 \mathrm{ml}$. volume of medium.

(ii) Air-pressure restoration vessel. When siphoning of medium is taking place the air pressure in the medium vessel tends to become lower than in the rest of the system owing to the faster flow of the medium. This differential pressure resulted in incomplete siphoning. The function of the pressure-restoration vessel is to store the medium 
for a few seconds and let the pressure equilibrate between its headspace and the metering vessel. This was not feasible with tubular connexions. The medium then enters the culture vessel.

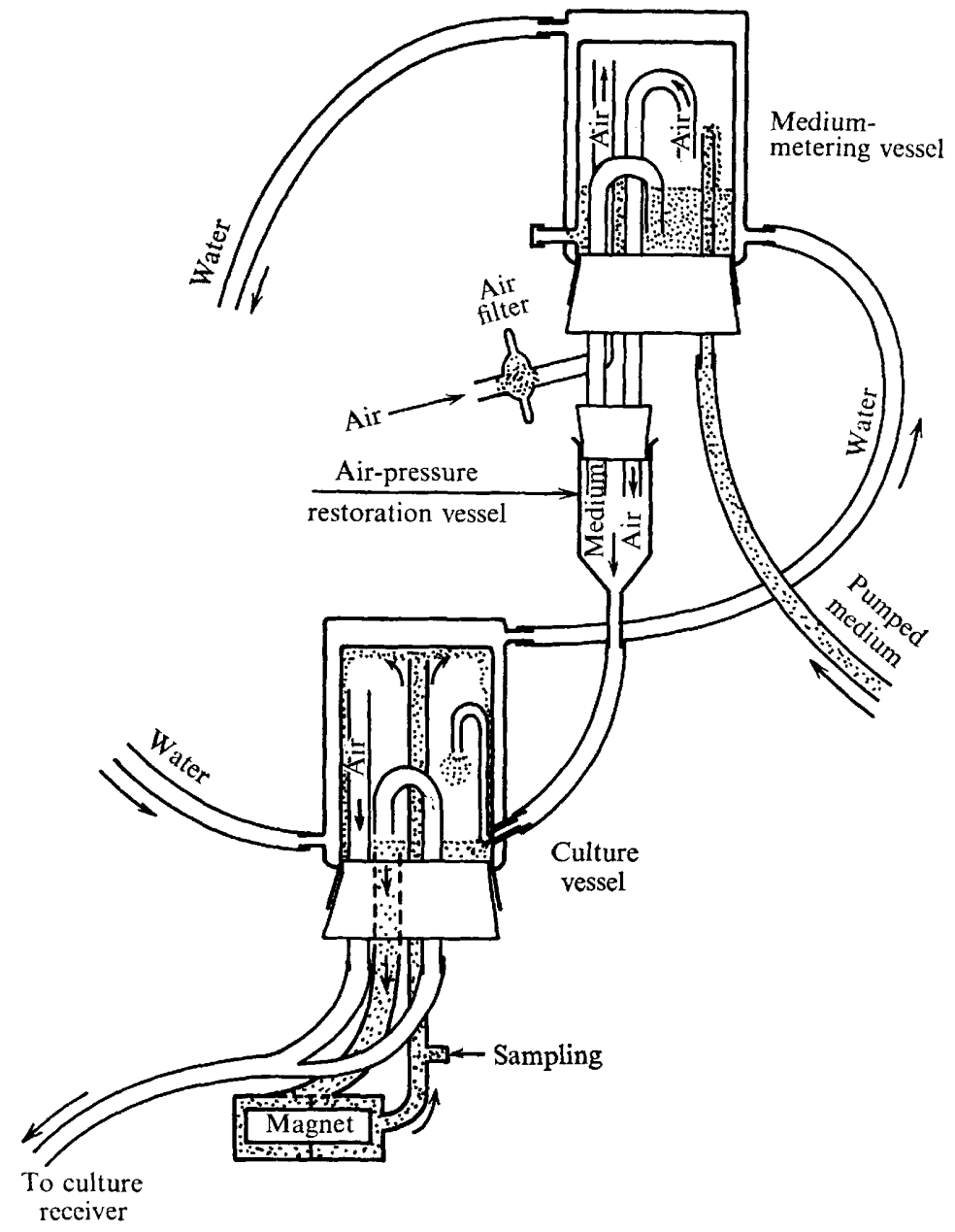

Fig. I. Drawing showing structural details of the culture system.

(iii) Culture vessel. This is similar to the medium metering vessel. The incoming air and medium enter the vessel through the original overflow of the vessel and a glass tube attachment bent upwards and then downwards at $180^{\circ}$ over the level of the $100 \mathrm{ml}$. mixture. By this arrangement, bubbling of air into the culture and frothing are avoided. This narrow-bored siphon-like tube has also the function of ensuring a prompt operation of the culture siphon by partially obstructing the free flow of both medium and air; this creates an increased air pressure behind the medium stream, and its release, when all medium has flown into the vessel, triggers the siphoning action. This happens within 3 to $4 \mathrm{sec}$. from the beginning of inflow of medium. Complete mixing of culture and fresh medium therefore becomes essential, and since no suitable pump existed a special pump was designed. The air outlet of the culture vessel joins the siphon outside the vessel on its way to the culture receiver. 
(iv) Culture pump. Another function of the magnetic pump is to aerate the culture. The outlet of the pump enters the culture vessel from the centre of the rubber stopper and its opening is just underneath the ceiling of the culture vessel. The culture is pumped against the ceiling and forms a thin film descending the side walls of the vessel, offering a large surface for efficient aeration. On the outlet, outside the culture vessel, there is a sampling point and a side connexion (not shown in Fig. I) to the optical density attachment. The inlet is in the centre of the top of the pump and its opening is at the level of the rubber stopper and well below the surface of the culture.

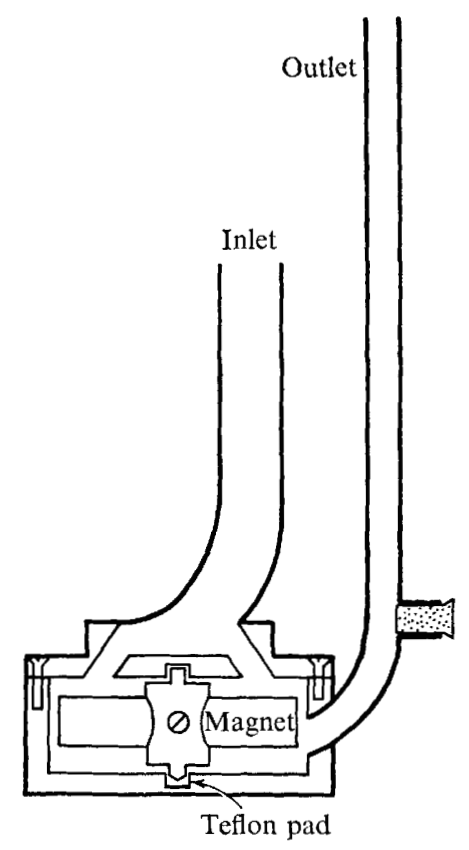

Fig. 2. The magnetic pump-mixer.

The pump (Fig. 2) is essentially of a centrifugal type; it consists of a stainless-steel case enclosing a magnet rotating round a fixed centre and gives a satisfactory output $(\mathrm{I} \cdot 5 \mathrm{l} . / \mathrm{min}$. and a $35 \mathrm{~cm}$. pump head). Inlet and outlet of pump, culture siphon, air outlet and rubber stopper form a firm assembly plugged into the culture vessel. More details can be seen in Fig. $I$.

With an initial volume of medium of 181 . the system operates continuously for periods ranging from 15 days ( $\mathrm{h}$. cycles) to 2 months ( $4 \mathrm{~h}$. cycles) although this period can be extended considerably, and this is our practice, by using intermittent operation provided the culture is allowed each time to become phased.

Assembly of the system, medium supply and sterilization. There are three openings in the system: (a) the air inlet of the medium metering vessel, which is an air filter; (b) the air-culture outlet of the culture vessel, which is connected with the culture receiver; $(c)$ the medium inlet of the medium metering vessel, which is connected to the medium pump tube and the medium supply vessel (Fig. 3).

The medium supply vessel is calibrated so that the appropriate volume of sugar solution from the respective reservoir is calculated and added to the medium. The 
sugar solution is first measured in a burette attached to the system and then forced into the supply vessel. The openings of the sugar vessel, burette and medium supply vessel are protected by air filters, and the whole system, medium supply and phasing apparatus is autoclavable as one unit.

Setting up the culture system. The phasing system was first connected to the air supply line at a flow rate $0 \cdot 31 . / \mathrm{min}$. Preliminary work with lower and higher flow rates showed that $0.31 . / \mathrm{min}$. results both in efficient oxygenation of the culture and satisfactory conditions for the automatic operation of the system.

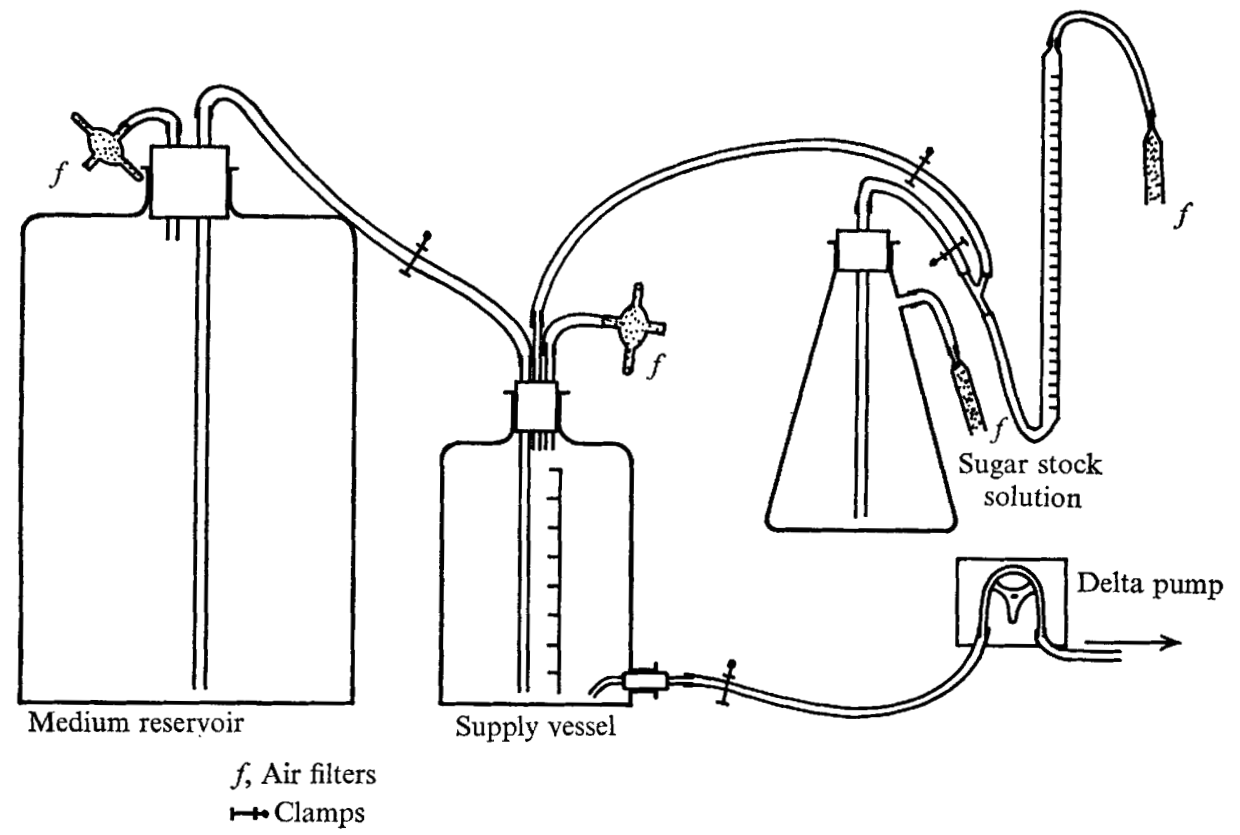

Fig. 3. The medium supply system.

The medium pump was controlled by a time-switch adjusted to a $50 \mathrm{ml}$. supply every $66 \mathrm{~min} ., 2 \mathrm{~h}$. or $4 \mathrm{~h}$. The temperature of the system was maintained at $37^{\circ}$. For the inoculation of the $50 \mathrm{ml}$. medium in the culture vessel, $1 \mathrm{ml}$. of an overnight shaken culture was injected from the sampling point of the system. The culture was allowed to reach the stationary phase before periodic operation of the system commenced.

Bacterial culture. A strain of Escherichia coli в 5 from our stock collection was used throughout these experiments. The generation time in the shaken flask at $37^{\circ}$ was about $50 \mathrm{~min}$.

Culture medium. A defined salt solution, at $\mathrm{pH} 7 \cdot 4$, was used as the growth medium for both inoculum and phased culture. The medium contained the following constituents (g./l.): $\mathrm{K}_{2} \mathrm{HPO}_{4}, \mathrm{II} \cdot 3 ; \mathrm{NaH}_{2} \mathrm{PO}_{4} \cdot 2 \mathrm{H}_{2} \mathrm{O}, 5 \cdot 4 ; \mathrm{MgSO}_{4} \cdot 7 \mathrm{H}_{2} \mathrm{O}, 2 \cdot 0 ; \mathrm{CaCl}_{2}$, O.OI ; $\mathrm{FeSO}_{4} \cdot 7 \mathrm{H}_{2} \mathrm{O}, 0.005 ; \mathrm{ZnSO}_{4} \cdot 7 \mathrm{H}_{2} \mathrm{O}, 0.0005 ; \mathrm{MnSO}_{4} .4 \mathrm{H}_{2} \mathrm{O}, 0.0005 ; \mathrm{CuSO}_{4} \cdot 5 \mathrm{H}_{2} \mathrm{O}$, $0.000 \mathrm{I}$; $\mathrm{CoCl}_{2} .6 \mathrm{H}_{2} \mathrm{O}$, 0.000I; sodium borate, $0.000 \mathrm{I}$; sodium molybdate, $0.000 \mathrm{I}$; ethylene-diaminetetra-acetic acid (EDTA), $0 \cdot 26 ; \mathrm{NH}_{4} \mathrm{Cl}, 2 \cdot 0$; glucose was the growthlimiting factor and its concentration was separately specified. The preparation of the medium was described by Pirt (1967). 
Examination of cultures. Optical density (O.D.) readings were made in an EEL colorimeter. The culture was allowed to flow into the o.D. tube attached to the system at a point on the circulation-pump outlet. After reading, the culture was forced back into the vessel by means of filtered air under pressure.

Culture samples, 0.2 to $0.3 \mathrm{ml}$., were withdrawn by means of a syringe and assayed immediately for viable and/or total counts. Viable counts were determined after incubation on Oxoid nutrient agar at $30^{\circ}$ for $24 \mathrm{~h}$. A dilution procedure described elsewhere (Anagnostopoulos, 1970) was used because it required only a small amount of culture and was quick and efficient. Dilutions were made in saline and the nutrient agar plates were surface-inoculated in quintuplicate with $0.02 \mathrm{ml}$. drops of an appropriate dilution; a 50 drops $/ \mathrm{ml}$. pipette was used for this. Counting within drop areas containing more than 30 colonies was made easier by the use of an enlarger with a projection screen (Shadomaster, Buck and Hickman, Otterspool Way, Watford, Hertfordshire).

Total counts on undiluted culture samples were made in a Helber counting chamber. The bacteria were allowed to settle for Io min. before counting, and the mean of 80 squares of four equidistant rows was taken as the total count. All cells were counted, but this was not thought to introduce any considerable error as the culture was mostly in a single-cell state and only occasionally were pairs of cells seen separated by less than their own length.

\section{RESULTS AND DISCUSSION}

An overnight culture of Escherichia coli inoculated into the culture vessel was allowed to reach the stationary phase, and the cyclic operation of the system started with a cycle length just over $\mathrm{I} h$. The glucose concentration of the medium supply was $0.1 \%(\mathrm{w} / \mathrm{v})$, which became $0.05 \%$ in the culture vessel at the beginning of each cycle plus the remaining sugar, if any, from the previous cycle. The semi-continuous growth of the culture was followed by periodical o.D. readings and occasional viable counts over 3 days of growth, i.e. over about 60 cycles. The results appear in Fig. 4 and 5 . A developing change in the rate of biomass increase within the cycles became noticeable, especially after $\mathrm{I} 8 \mathrm{~h}$. (Fig. $4 \mathrm{~b}$ ). On the third day the o.D. curve attained an almost bi-rectilinear form (Fig. 5). Viable counts (Fig. $4 b, 5$ ) showed that the above changes were accompanied by synchronization of division.

The bi-rectilinear form of the o.D. curve was initially thought to represent a settled condition of the culture so that this should repeat itself indefinitely, thereby providing a permanent supply of phased culture. It was of interest to see the response of a different inoculum of the same bacterial culture at the same glucose level in a longer $2 \mathrm{~h}$. cycle. Six days after inoculation the first o.D. observations showed also the bi-rectilinear form of O.D. curves previously observed with the I h. cycles. Nevertheless, the intersections of the two components of each of a series of consecutive curves (Fig. $6 a$ ) were not timed equally from the origin of the curve as was thought to be the case with the I h. cycles (Fig. 5); also, the slopes of the respective component straight lines varied between different cycles. In addition, the same culture I day later showed a diauxie curve (Fig. $6 \mathrm{~b}$ ). This observation pointed towards a further wave of biomass synthesis which could be due to utilization of metabolic products as carbon sources following the exhaustion of glucose. The intersections of the bi-rectilinear curves might 
well be indicative of a critical low level of glucose, and this is under investigation. Furthermore, this burst of growth suggests the release of metabolites by bacteria losing viability which are being utilized by surviving bacteria. Loss of viability had been observed in early experiments and was attributed to technical errors; nevertheless, loss of viability subsequently proved to be an important feature of this system. Examples are seen in Fig. 7, 8 and 9, representing I, 2 and 4 h. cycles respectively. A stepwise restoration of the initial numbers and a further doubling is clearly noticed

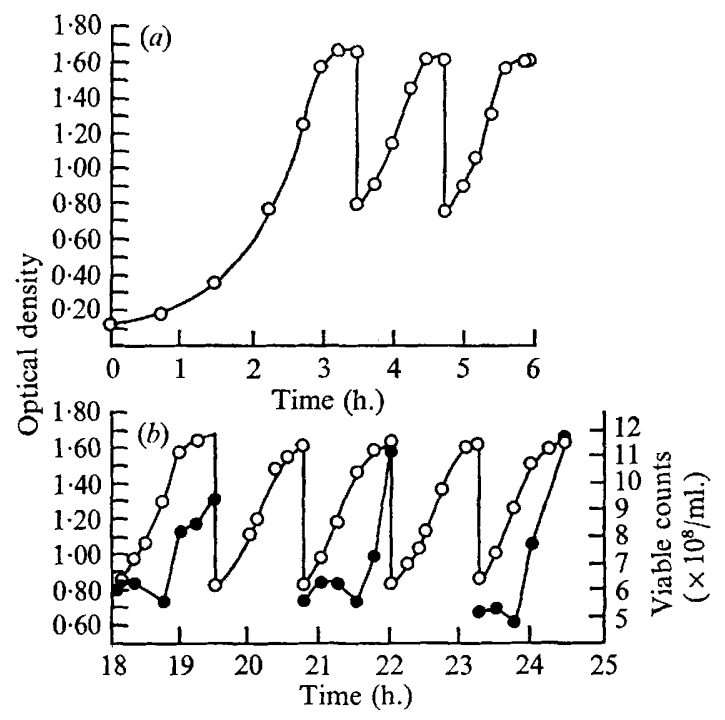

Fig. 4

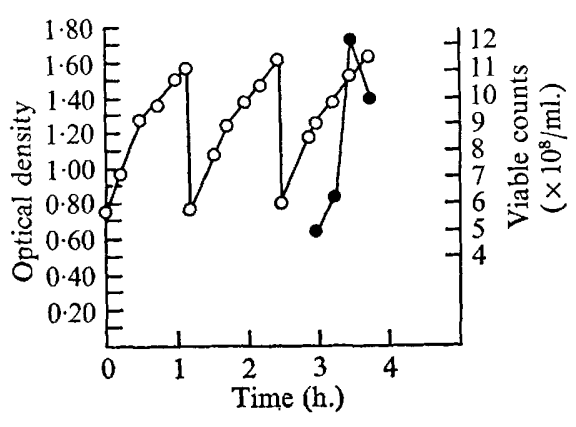

Fig. 5

Fig. 4. One h. cycle. (a) Initiation of semi-continuous growth; (b) growth as observed over 5 cycles $18 \mathrm{~h}$. later. The transition from exponential growth to plateau, rapid with building blocks such as glucose when the concentration falls below a minimum, becomes gradual as the cyclic growth proceeds. Viable counts show the first signs of synchronization. $\bigcirc-O$, Optical density; - viable counts.

Fig. 5. One h. cycle. The same culture as in Fig. 4 on it third day of growth. The o.D. curves became uniformly bi-rectilinear, with their intersections occurring towards the middle of the cycles. A synchronous division is also shown. $\mathrm{O}-\mathrm{O}$, Optical density; - , viable counts.

in Fig. 9. Total counts may also be seen in Fig. 7(a) and Fig. Io, where a doubling of the whole population follows a period equal to about three-quarters of the cycle. Another characteristic of the system is that the initial decline in viability is exponential in nature and reaches the lowest value about $\mathrm{I} h$. from the beginning of both the 2 and 4 h. cycles. What happened in two consecutive $\mathrm{I} \mathrm{h}$. cycles is depicted in Fig. 7. Here we observe the initial decline followed by an increase to double the viable count in part (a); after siphoning out of half of the culture medium the culture seems to consist of two growing components dividing synchronously in part $(b)$. In part $(a)$ the cycle begins with $4.75 \times 10^{8} / \mathrm{ml}$. viable counts and $7 \cdot 2 \times 10^{8} / \mathrm{ml}$. total counts. The difference of $2.4 \times 10^{8} / \mathrm{ml}$. must represent non-viable cells carried over from the previous cycle. The viable population declined in the first 15 min. to a mean $3.65 \times 10^{8} / \mathrm{ml}$. and after 50 to $60 \mathrm{~min}$. divided synchronously to increase both the viable and total counts 


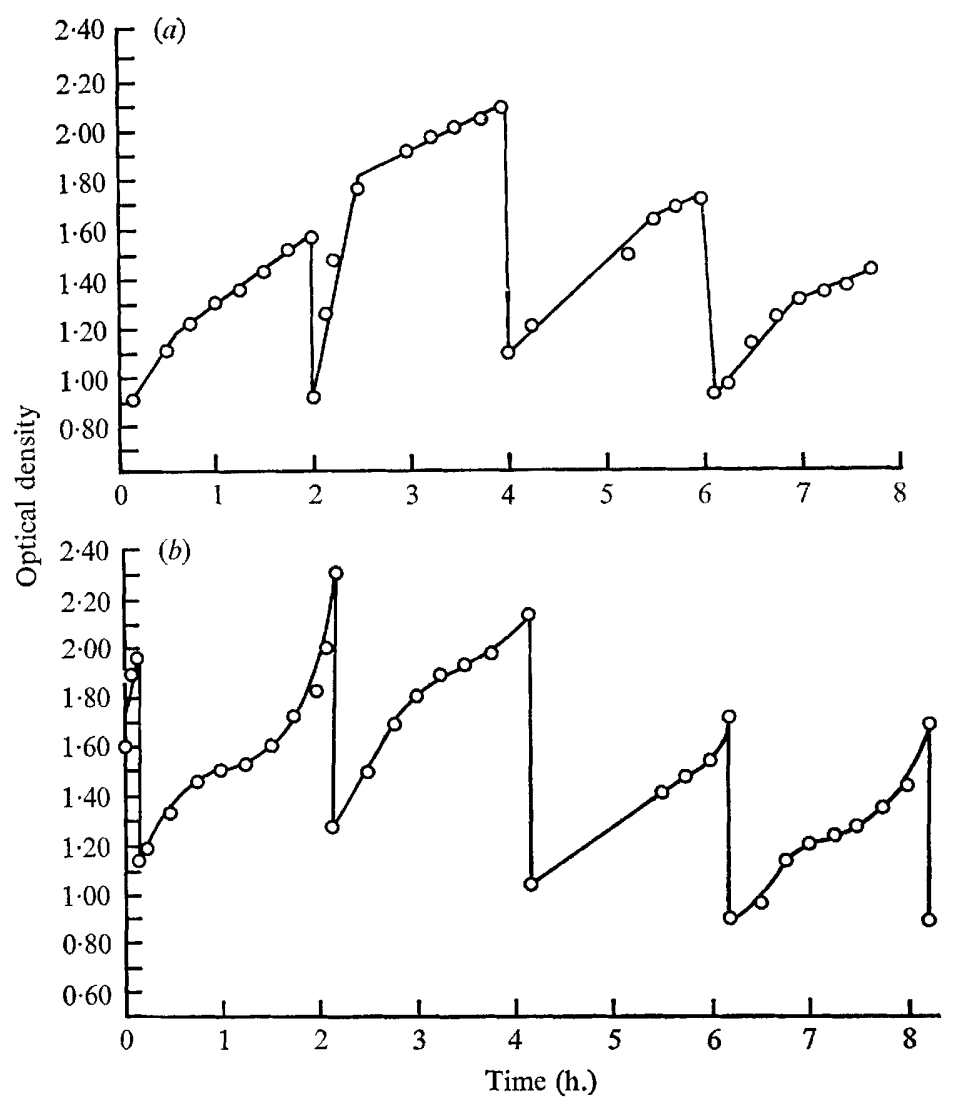

Fig. 6. Two h. cycle. (a) Bi-rectilinear o.D. curves of a 6 day culture; they lack the uniformity of Fig. 5. (b) The diauxie pattern of growth observed the following day.

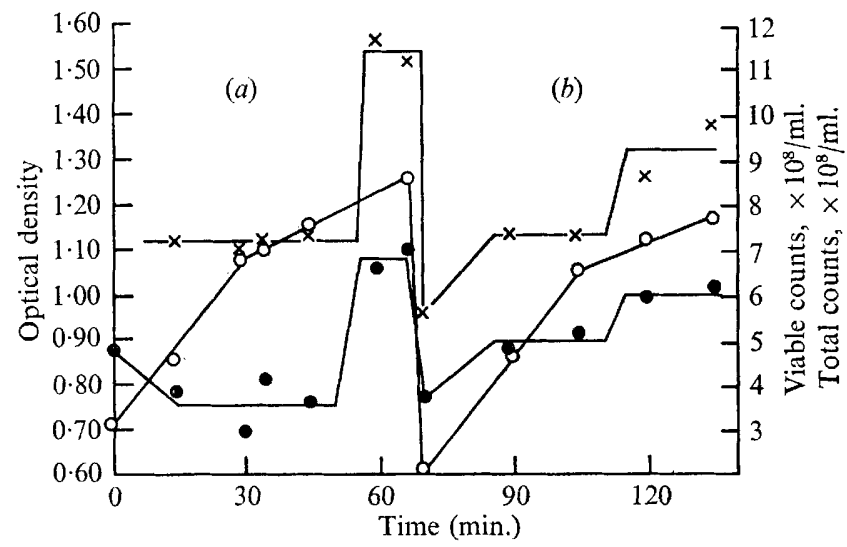

Fig. 7. One h. cycles. In part (a), after an initial loss of viability, the culture doubled itself synchronously. In part $(b)$ the newly divided population consisted of two synchronously but independently dividing fractions. The biomass synthesis shows the familiar bi-rectilinear o.D. curves. The high total counts as compared with the viable counts are due to non-viable cells carried over from the previous cycle (see text). - Viable counts; $\times-x$, total counts; $\mathrm{O}-\mathrm{O}$, optical density. 
by almost the same amount to $6.8 \times 10^{8}$ and $\mathrm{II} \cdot 5 \times 10^{8} / \mathrm{ml}$. respectively. Cycle $(b)$ was entirely different. This underlines another feature of the system - that no two consecutive cycles were identical with respect to total counts, viable counts and optical density. This is against the view of Dawson and his colleagues (Dawson, 1965; Müller \& Dawson, I968; Dawson \& Kurz, 1969) that each cycle repeats itself indefinitely thereby providing a reproducible system of synchronized bacterial populations. On siphoning

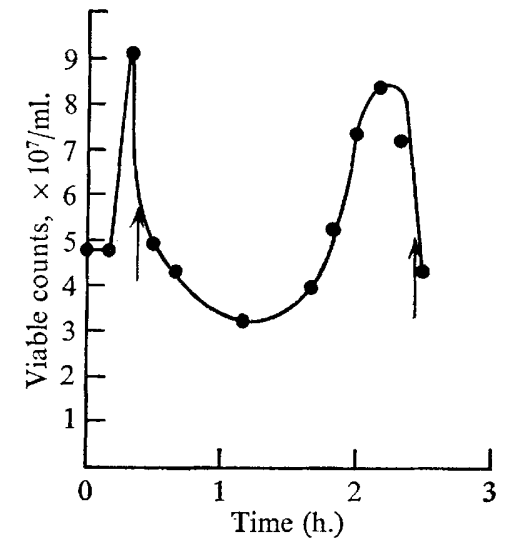

Fig. 8

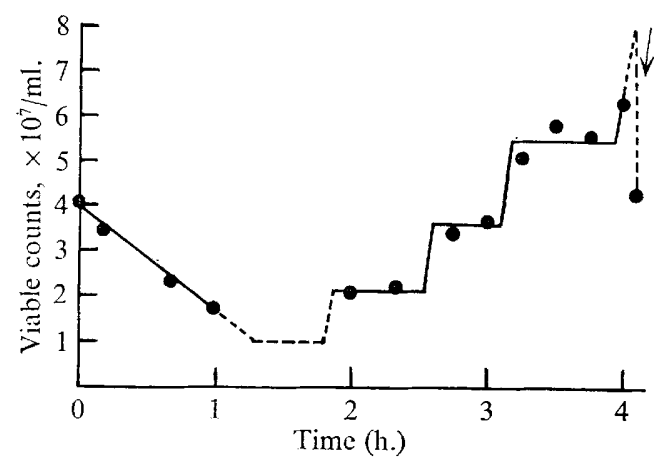

Fig. 9

Fig. 8. Two h. cycle. Viable counts show a perfect synchronous division followed, on introduction of fresh medium, by loss of viability, restoration of initial numbers and a further synchronous division towards the last quarter of the cycle. The arrows indicate the time of siphoning.

Fig. 9. Four h. cycle. The initial decline in viable counts was followed by successive fractional synchronous divisions to a final number double that at zero time, as shown by the viable count following siphoning (arrow). - - , Assumed counts.

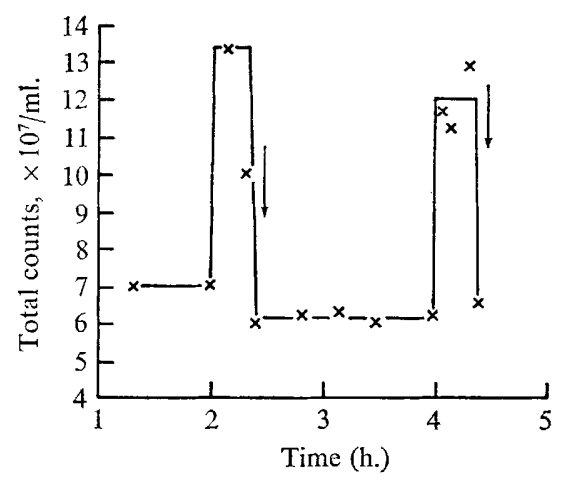

Fig. 10

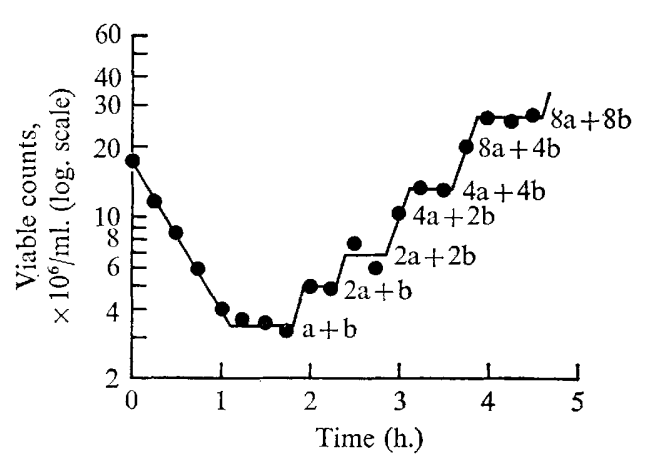

Fig. I I

Fig. 10. Two h. cycle. Total counts showing synchronized division in the last quarter of the cycle. The existence of a plateau over a period of $\mathbf{I} \cdot 5 \mathrm{~h}$. before doubling can only mean that the cells which lost their viability and were replaced through continued division of the viable population (see Fig. 8,9 ) gradually underwent lysis. The arrows indicate the time of siphoning.

Fig. I I. Culture from a $4 \mathrm{~h}$. cycle transferred just after its dilution into a flask and shaken (at $37^{\circ}$ in excess glucose). Viable counts illustrate two features of the semi-continuous system: the logarithmic loss of viability at the beginning of the cycle already observed, and the existence of two synchronously but independently dividing populations, $a$ and $b$. 
after $70 \mathrm{~min}$. the increase in the non-viable population $\left(3.5 \times 10^{8} / \mathrm{ml}\right.$. - the total counts less the viable counts) was halved to $1.8 \times 10^{8} / \mathrm{ml}$. The corresponding total and viable counts were $5.6 \times 10^{8}$ and $3.75 \times 10^{8} / \mathrm{ml}$. The division in part $(b)$ occurred in two steps. First, $50 \%$ of the population divided $30 \mathrm{~min}$. after the previous division, i.e. in less than the normal generation time. Released metabolites from non-viable cells may have been responsible for this. The second half showed the normal generation time. The disproportional increase of the total counts at each division reveals that some of the newly divided bacteria became non-viable. The difference in generation time of the two parts of the population, both products of the previous synchronous division, should certainly be related to that division. One is tempted to speculate that $50 \%$ of the offspring daughter cells were more competent than the rest to speed up their division cycle under the influence of growth-promoting factors released by the non-viable bacteria carried over from the previous cycle.

This is better demonstrated when the culture at the beginning of a $4 \mathrm{~h}$. cycle is transferred into medium with excess glucose in a flask shaken at $37^{\circ}$ (Fig. II). After an $80 \%$ decrease in the viable population within the first hour of subculture, two equal parts of the population appeared as before and divided synchronously but at different times. The successive divisions of the two equal fractions $a$ and $b$ proceeded as follows (viable counts $\times 10^{7}$ in parentheses:)

$$
\begin{gathered}
\mathrm{a}+\mathrm{b}(0.34)-2 \mathrm{a}+\mathrm{b}(0.50)-2 \mathrm{a}+2 \mathrm{~b}(0.68)-4 \mathrm{a}+2 \mathrm{~b}(\mathrm{I} \cdot 02)-4 \mathrm{a}+4 \mathrm{~b}(\mathrm{I} \cdot 30) \\
8 \mathrm{a}+4 \mathrm{~b}(\mathrm{I} \cdot 99)-8 \mathrm{a}+8 \mathrm{~b}(2 \cdot 67) .
\end{gathered}
$$

The overlapping division cycles eventually coincided (G. D. Anagnostopoulos, unpublished results) to give a wholly synchronized culture after as many as I4 fractional divisions.

From these results it seems that during successive cycles of the phasing system part of the emerging population from a division loses its viability in a logarithmic fashion on introduction of the fresh medium. At that stage biomass synthesis is at its maximum as shown by the increase in optical density. It seems therefore that unbalanced growth is responsible for the loss of viability. This effect occurs under conditions where cycles are longer than the normal generation time of the organism. Dilution of cultures with equal volumes of fresh medium at intervals equal to the generation time of an organism is a technique for maintaining balanced growth (Schaechter, Maaløe \& Kjeldgaard, 1958). Therefore, the unbalanced growth observed in our system may reflect a defective post-division state of the division mechanism of the emerging population leading to loss of viability under conditions favourable for growth and these observations may be related to the tendency of cultures in the transition period between logarithmic and stationary phase of growth to become synchronized on transfer into fresh medium.

I thank Professor S. J. Pirt for his helpful criticism during the course of this work and Dr A. P. J. Trinci for his advice in the preparation of the manuscript.

\section{REFERENCES}

Anagnostopoulos, G. D. (1970). A rapid dilution technique. Journal of General Microbiology $64,25 \mathrm{I}-252$.

CUTLER, R. G. \& Evans, J. E. (1966). Synchronization of bacteria by a stationary-phase method. Journal of Bacteriology 9r, 469-476. 


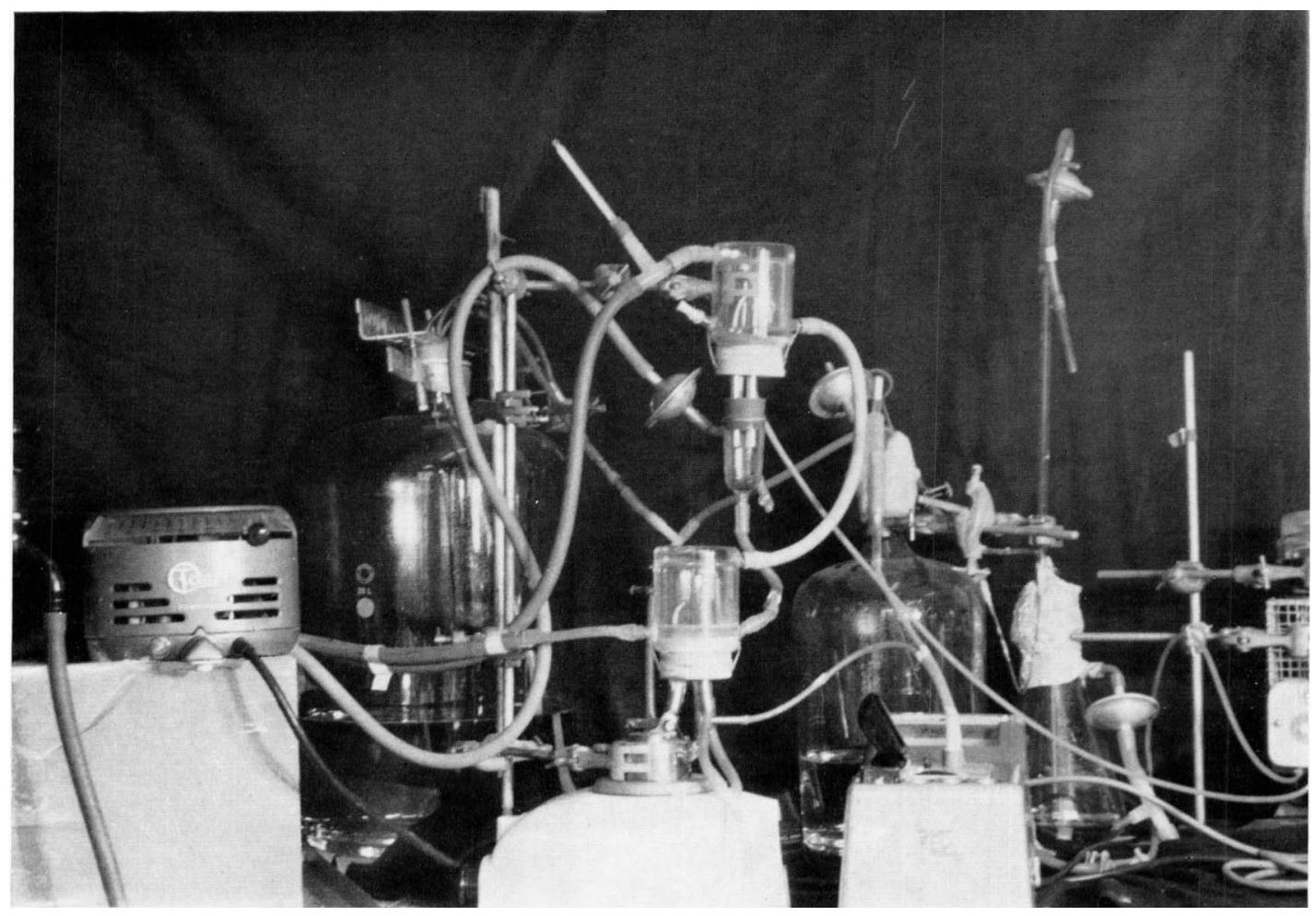


DAwson, P. S. S. (1965). Continuous phased growth, with a modified chemostat. Canadian Journal of Microbiology II, 893-903.

Dawson, P. S. S. \& KURz, W. G. W. (1969). Continuous phased cultures - a technique for growing, analysing and using microbial cells. Biotechnology and Bioengineering II, 843-85I.

Müller, J. \& Dawson, P. S. S. (I968). The operational flexibility of the phased culture technique, as observed by changes in the cell cycle of Candida utilis. Canadian Journal of Microbiology r4, I I I 5-I I 26.

Nishi, A., OKamura, S. \& Yanagita, T. (1967). Shift of cell-age distribution pattern in the later phases of Escherichia coli cultures. Journal of General and Applied Microbiology 13, 103-119.

PIRT, S. J. (1967). A kinetic study of the mode of growth of surface colonies of bacteria and fungi. Journal of General Microbiology 47, 18 I-197.

PIRT, S. J. \& Callow, D. S. (1964). Continuous flow culture of the ERK and L types of mammalian cells. Experimental Cell Research 33, 413-421.

Schaechter, M., MaAløe, O. \& KueldgaARd, N. O. (1958). Dependency on medium and temperature of cell size and chemical composition during balanced growth of Salmonella typhimurium. Journal of General Microbiology 19, 592-606.

\section{EXPLANATION OF PLATE}

The phasing culture system, showing the medium and culture vessels, the magnetic pump and the o.D. attachment. The overall height of the apparatus is $45 \mathrm{~cm}$. 